\title{
Evitar errores a veces compete a los profesionales, a veces a los pacientes, pero a veces a los directivos
}

\section{Sometimes avoiding errors is the responsibility of professionals, at others of patients, but sometimes it falls to managers}

\section{J.J. Mira Solves ${ }^{1}$}

\section{Sr Director:}

Aunque lo más habitual cuando se produce un error es atribuir a un profesional la responsabilidad y la culpa, se ha puesto de manifesto que los fallos de sistema que comprometen a una parte de la organización son mucho más frecuentes que el error humano.

Los modelos que analizan el error, suelen aludir a ellos como error atribuible a las relación persona-contexto ${ }^{1}$. Estos fallos de sistema tienen una variada casuística aunque la carga de trabajo y las condiciones del contexto donde se desarrolla la actividad han sido los aspectos más estudiados.

En un clásico estudio de la $\mathrm{NASA}^{2}$ de 1993 se puso sobre el tapete una realidad que se sospechaba. Cuando existe una excesiva carga de trabajo (hurry-up-syndrome)es más fácil que se produzcan accidentes (hasta un $80 \%$ de los accidentes aparecieron relacionados con una excesiva carga de trabajo). Las condiciones de ambiente, entorno y otros factores organizativos, como estilos de dirección y supervisión, han sido analizados también en relación a los errores en el sector del transporte, aéreos, marítimos o ferroviarios ${ }^{1}$. En el sector sanitario este tipo de estudios son mucho menos frecuentes pese a que intuitivamente podamos sospechar que debe existir relación entre ambos.

La Fundación de la Seguridad del Paciente $^{3}$ en EEUU sugirió en 2002 que a la hora de analizar los factores que inciden en el riesgo de que se produzca un error deben considerarse:

- Factores del paciente

- Factores de la tarea a realizar

- Factores individuales del personal

- Factores del equipo de personal

- Ambiente de trabajo

- Organización y administración

- Contexto institucional

En un reciente trabajo publicado en esta revista titulado: Factores del entorno de trabajo que influyen en la ocurrenciade errores de administración de medicación, Ambrosio y Pumar-Méndez ${ }^{4}$ revisan la literatura en relación a errores en la administración de medicación que pueden ser atribuidos a factores del entorno de trabajo. En este trabajo, donde se revisa un total de 8 estudios publicados entre 2002 y 2012 , se concluyó que entre los precursores de los errores en la administración de medicación se hallaban las distracciones, las interrupciones, y la sobrecarga de trabajo. Es interesante en este trabajo la sucesiva relación de propuestas para incrementar la seguridad y evitar el efecto de distracciones e interrupciones y estrategias para evitar confusiones asociadas a una mayor presión o carga de trabajo.

El objetivo del estudio no permitió analizar otros factores que también inciden en la ocurrencia de errores y que deben tenerse en cuenta. Por ejemplo, los relacionados con la inexperiencia asociada al inicio de la 
actividad profesional y los achacables a un descanso inadecuado de los profesionales antes de la jornada de trabajo.

El estudio de Volpp y Grande ${ }^{5}$ relaciona una serie de propuestas para evitar los errores de los residentes, especialmente, cuando inician la residencia. Este tipo de recomendaciones cabe extenderlas a otros profesionales cuando por rotaciones, cambios de plantilla o tomas de posesión se incorporan a un servicio o una unidad diferente a la habitual.

Por su parte, Rothschild y $\operatorname{col}^{6}$ en su análisis de la influencia de las horas de trabajo y las horas de sueño el día antes a entrar en quirófano inciden en la frecuencia de complicaciones durante las intervenciones hallaron que mientras que la duración de la jornada no suponía un mayor riesgo de ocurrencia de complicaciones durante la intervención, un descanso menor a 6 horas durante la noche no era recomendable para cirujanos y ginecólogos que tenían programadas intervenciones al día siguiente. En su análisis de los resultados en cinco procesos concretos realizados por un total 86 cirujanos y 134 ginecólogos, hallaron que cuando el médico había descansado 6 o menos horas, aparecieron complicaciones en el $6,2 \%$ de las intervenciones deprimera hora de la mañana, mientras que si el descanso era superior a 6 horas este porcentaje descendía hasta el 3,4\% (OddsRatio,1,72; IC 95\%, 1,02-2,89).

A la preocupación por atrapar el error hemos incorporado medidas para evitarlos en el ámbito de los procedimientos y de las técnicas aplicadas y los profesionales, en el ámbito de sus respectivos servicios y unidades, han liderado procedimientos que incrementan la seguridad de los pacientes. Se ha analizado el papel de los pacientes como segundo control para reducir eventos adversos ${ }^{7}$, los errores de los propios pacientes $^{8} \mathrm{y}$ desarrollado procedimientos y herramientas para ayudarlesa evitarlos en la medida de lo posible ${ }^{9}$. No obstante, hay otros factores de riesgo para la seguridad que dependen de decisiones organizacionales que se adoptan en el ámbito directivo y que en un contexto de restricción económica como el actual podemos tener la tentación de relegar a un segundo plano. El primero, dejar de lado los esfuerzos por promover una cultura proactiva de seguridad que busque implantar medidas con las que adelantarse a los riesgos para los pacientes (y los propios profesionales). Segundo, no reconocer a tiempo que el ambiente, contexto y aspectos organizativos, como los descritos en estos estudios, inciden en los riesgos para la seguridad de los pacientes.

Es cierto que los profesionales tienen la responsabilidad de asegurarse de que funcionan las barreras a la ocurrencia para evitar eventos adversos funcionan y deben incorporar otras nuevas. También que los pacientes deben participar de una forma más activa para contribuir a evitar errores. Pero no debemos olvidar que la implicación de los directivos es también clave para lograr un entorno más seguro y algunas pistas sobre qué requiere su atención ya las vamos teniendo.

\section{BIBLIOGRAFÍA}

1. Sebastián ML. Fallo humano: la quiebra de un paradigma. Apuntes de Psicología 2009; 27: 21-51.

2. NASA. ASRS (Aviation Safety Reporting System). Disponible en: http://asrs.arc.nasa.gov/publications/directline/dl5_hurry.htm. Consultado 25-52013.

3. Focus on Patient Safety. NPSF. 2002: 5 (2).

4. Ambrosio L, Pumar-Méndez MJ. Factores del entorno de trabajo que influyen en la ocurrencia de errores de administración de medicación. An Sist Sanit Navar 2013; 36: 77-85.

5. Volpp KGM, Grande D. Patient Safety: Residents' Suggestions for Reducing Errors in Teaching Hospitals N Engl J Med 2003; 348: 851-855.

6. RothsChiLd JM, Keohane CA, Rogers S, GardnER R, LiPSITZ SR, SALZBERG CA et al. Risks of complications by attending physicians after performing nighttime procedures. JAMA 2009; 302: 1565-1572.

7. SсHWAPPACH DL. Review: engaging patients as vigilant partners in safety: a systematic review. Med Care Res Rev 2010; 67: 119-148.

8. Mira JJ, Navarro IM, Guilabert M, Aranaz J. Frecuencia de errores de los pacientes con su medicación. Rev Panam Salud Publica 2012; 31: 95-101.

9. Botella F, Borras F, Mira JJ. Safer Virtual Pillbox: Assuring Medication Adherence to Elderly Patients. En: Proceedings of the Third ACM MobiHoc Workshop on Pervasive Wireless Healthcare, MobileHealth-2013, Bangalore, India, July 29-Ago 1, 2013. ACM Press. New York, NY, USA (C2013. 